\title{
Cognição social na esquizofrenia: um enfoque em habilidades teoria da mente
}

\section{Social cognition in schizophrenia: focus on theory of mind abilities}

\author{
Helio Tonelli'; Cristiano Estevez Alvarez"I \\ 'Mestre, Farmacologia, Universidade Federal do Paraná (UFPR), Curitiba, PR. Psiquiatra do Instituto \\ de Psiquiatria do Paraná (IPP), Curitiba, PR. \\ "Mestrando, Psiquiatria, Universidade Federal de São Paulo (UNIFESP), São Paulo, SP. Psiquiatra, \\ Instituto de Psiquiatria do Paraná (IPP), Curitiba, PR. \\ Endereço para correspondência
}

\section{RESUMO}

"Teoria da mente" é o nome que tem sido dado à habilidade que os seres humanos têm de inferir os estados mentais ou as intenções de outros seres humanos. Tais habilidades fazem parte de um grupo maior de capacidades cognitivas, especificamente relacionadas ao comportamento social, denominado cognição social. A esquizofrenia é um transtorno mental que costuma cursar grave comprometimento do funcionamento social. Existem vários estudos correlacionando transtornos das habilidades teoria da mente e sintomas da esquizofrenia com resultados ainda controversos. Muitos autores acreditam que os sintomas da esquizofrenia podem ser diretamente compreendidos à luz de alterações das habilidades teoria da mente, enquanto outros argumentam que as alterações dessas habilidades observadas em esquizofrênicos são reflexo de seu comprometimento cognitivo geral. Ainda existem poucos estudos relacionando o impacto do uso de antipsicóticos sobre a cognição social e habilidades teoria da mente e eles apresentam problemas metodológicos.

Descritores: Cognição, esquizofrenia, transtornos psicóticos.

\begin{abstract}
"Theory of mind" is the term used to designate human abilities to infer the state of mind or intentions of others. Such abilities are part of a major group of cognitive capabilities specifically related to social behavior, known as social cognition. Schizophrenia is a mental disorder that usually consists of severe social functioning. There are many studies available on the relations between disorders of theory of mind abilities and schizophrenia symptoms. Many authors believe that schizophrenia symptoms might be directly understood by focusing on some changes in Theory of Mind abilities. Other authors claim that such changes observed in schizophrenic patients are the result of their general cognitive impairment. There are still few studies related to the impact of the use of antipsychotics on social cognition and Theory of Mind abilities, which present methodological problems.
\end{abstract}

Keywords: Cognition, schizophrenia, psychotic disorders. 


\section{NTRODUÇÃO}

O comprometimento no funcionamento social é um importante sintoma da esquizofrenia e pode ocorrer tanto na forma de isolamento social quanto na forma de inadequação afetiva e comportamental. Esse tipo de comprometimento, embora esteja também presente em outros transtornos mentais, é muito mais marcante na esquizofrenia, podendo surgir ao longo de todo o curso do transtorno, persistir a despeito do tratamento antipsicótico, tendendo à estabilidade ou mesmo à piora com a evolução do quadro. Além disso, o prejuízo do funcionamento social aumenta as taxas de recaída da esquizofrenia, tornando imprescindível o desenvolvimento de intervenções que melhorem essas funções ${ }^{1}$.

Embora se admita intuitivamente uma forte relação entre integridade neurocognitiva e adequação do comportamento social na esquizofrenia, as evidências disponíveis dando suporte a esta correlação são, ainda, modestas.

Por cognição social (CS) se subentendem "as operações mentais que estão por trás das interações sociais e que incluem a habilidade humana de perceber as intenções e disposições dos outros"1 ${ }^{\text {. As }}$ representações geradas por este tipo específico de cognição servem para que o indivíduo guie seu comportamento social de forma flexível e adaptada. Evidências de que a CS seja um domínio cognitivo separado resultam, por exemplo, de estudos de indivíduos que sofreram lesão cortical frontal ou pré-frontal, demonstrando que eles podem apresentar, como consequência dessas lesões, danos em seu comportamento social a despeito da manutenção de habilidades cognitivas como memória e linguagem².

Boa parte dos estudos a respeito da CS é desenvolvida a partir da observação de pacientes portadores de transtornos do espectro do autismo ${ }^{3,4}$, sendo muitas das conclusões desses estudos extrapoladas para pacientes esquizofrênicos. Nestes pacientes, o estudo da CS concentra-se, de maneira geral, no estudo das chamadas habilidades teoria da mente (também chamadas de "mentalização" e "metarrepresentação" ou, simplesmente, de "habilidades theory of mind (ToM)", expressão que será empregada neste texto), relacionadas à habilidade de representar os estados mentais e de fazer inferências acerca das intenções dos outros; e em estudos de percepção social (cujas linhas de pesquisa se concentram principalmente no reconhecimento do afeto facial e na percepção de atitudes sociais) e estilo atribucional ${ }^{5}$.

Este trabalho focalizará os estudos a respeito das habilidades ToM na esquizofrenia.

\section{MÉTODO}

Foi realizada pesquisa eletrônica na base de dados MEDLINE, sendo selecionados artigos nas línguas portuguesa e inglesa que incluíssem apenas estudos feitos com humanos, utilizando os descritores schizophrenia, psychotic disorders e antipsychotics associados à expressão theory of mind, sendo encontrados 77 artigos, dos quais foram selecionados aqueles diretamente relacionados ao tema em questão.

\section{Habilidades ToM}

Em 1978, dois primatologistas (Premack \& Woodruff) publicaram um artigo cujo título em português era "O chimpanzé tem uma teoria da mente?"6. Nele, os autores questionavam se o chimpanzé, a exemplo dos humanos, também poderia "perceber" que o comportamento dos seus companheiros era determinado por seus (dos companheiros) desejos. A expressão "habilidades ToM" deriva desse trabalho. Uma escola de pensamento associada a um autor chamado Frans de Waal assume que alguns grupos de primatas, particularmente os chimpanzés, apresentam rudimentos de comportamentos pró-sociais (como empatia, compaixão, moralidade e preocupação com outros seres da mesma espécie) derivados de habilidades $\operatorname{ToM}^{7}$. Há pesquisadores que não concordam com esta tese, argumentando que esses comportamentos pró-sociais não seriam universais e apenas apareceriam entre indivíduos da mesma família e nada teriam a ver com as habilidades pró-sociais 
desenvolvidas em seres humanos à custa da sofisticada evolução das habilidades ToM².

Apesar de as pesquisas a respeito do tema não terem sido favoráveis à possibilidade de os chimpanzés possuírem habilidades ToM² ${ }^{2}$ é certo que elas são uma importante e incontestável aquisição dos humanos. A espécie humana é altamente social. Nós, seres humanos, construímos e mantemos competentemente uma variedade de relações com outras pessoas. Essa competência dependeria da integridade de nossa CS e de nossas habilidades ToM, que descrevem nossa capacidade de inferir estados mentais de outros indivíduos, crenças, desejos e intenções e de apreciar o quanto esses estados mentais podem diferir dos nossos próprios.

Em relação à localização cerebral das habilidades ToM, os achados mais frequentemente replicados dos estudos de neuroimagem funcional e ToM envolvem o córtex pré-frontal, pois quase todas as tarefas que avaliam tais habilidades parecem ativar uma ou mais áreas dessa região ${ }^{8}$. No entanto, esses estudos mostram uma grande dispersão dos focos de ativação do córtex pré-frontal medial em indivíduos submetidos a tarefas ToM. Posto que o córtex pré-frontal também está relacionado a várias funções cognitivas gerais, como recuperação de memória episódica, processamento da linguagem e aprendizado de novas regras de resposta ${ }^{9}$, não é, obviamente, possível associar essa região exclusivamente às habilidades ToM.

\section{Testagem das habilidades ToM}

A avaliação das habilidades ToM tem sido feita usando-se testes compostos por tarefas envolvendo a descrição ou a ponderação sobre o conteúdo enganoso de recipientes ou a localização inesperada de objetos, bem como pequenas histórias em que é necessário colocar-se no lugar do protagonista ou fazer inferências a respeito dos estados mentais dos personagens. Estes testes podem, ainda, ser apresentados através de uma ampla variedade de formatos verbais ou ilustrações. No Sally Anne Task $^{10}$, por exemplo, é apresentada ao examinado a seguinte situação: dois personagens, Sally e Anne, possuem dois tipos de recipientes diferentes. Sally tem uma cesta, Anne tem uma caixa. Sally guarda um brinquedo dentro de sua cesta. Momentos depois, sem que Sally esteja presente, Anne aparece e tira o brinquedo da cesta de Sally e o coloca em sua caixa. O avaliador pergunta para o examinado onde Sally deverá procurar o seu brinquedo. O examinado terá testada sua habilidade de discernir, diante desta situação, que suas crenças diferem das de outras pessoas. $O$ fato de ele saber que o brinquedo foi trocado de lugar por Anne não implica em que Sally o saiba também. Não implica, portanto, que Sally vá buscar o brinquedo primeiro na caixa ao invés de na sua cesta. É testada, portanto, a capacidade do avaliado metarrepresentar o estado mental de Sally. Essa capacidade é também denominada "compreensão de uma falsa crença de primeira ordem" em contraste com falsas crenças mais sofisticadamente construídas e que incluem metáfora, ironia e faux pas ${ }^{11-13}$. O teste faux pas consiste em 10 histórias em que um dos personagens diz alguma coisa que não deveria ter dito. A história é lida em voz alta ao examinado, que deverá identificar se alguém disse algo que não deveria ter dito, bem como o personagem que o fez. Um exemplo de história faux pas seria: Jill acabou de se mudar para uma casa nova. Ela foi às compras com sua mãe e comprou cortinas novinhas em folha. Assim que Jill acabou de instalá-las, sua melhor amiga, Lisa, apareceu e disse: "Que cortinas horríveis, espero que você as troque logo..."14.

Baron-Cohen ${ }^{16,17}$ sugeriu que a interpretação do significado emocional do olhar de outros seres humanos também teria um papel importante no funcionamento de circuitos cerebrais supostamente envolvidos com o processamento da CS em humanos. No Eyes Test ${ }^{17}$ é apresentada ao participante uma série de 36 fotografias da região facial em torno de olhos humanos. Em um nível inconsciente, rápido e automático, o participante deve comparar a imagem dos fragmentos de rostos que observa nas gravuras que Ihe são apresentadas com os exemplos de expressões emocionais que tem armazenado na memória a fim de julgar qual é a melhor opção dentre as apresentadas. $O$ participante escolhe uma dentre quatro alternativas, a que melhor descreve o que a pessoa retratada estaria pensando ou sentindo.

De maneira geral, a avaliação objetiva das habilidades ToM ainda é pouco estudada, e as tarefas empregadas nos trabalhos a respeito das alterações dessas habilidades em esquizofrênicos, além de não terem sido desenvolvidas especificamente para eles, ainda não foram suficientemente validadas, razão pela qual todos esses estudos devem ser olhados com cautela ${ }^{18}$. 
Alterações das habilidades ToM na esquizofrenia

Existem muitos estudos a respeito de alterações da CS e das habilidades ToM em pacientes esquizofrênicos disponíveis na literatura internacional. Os estudos brasileiros acerca do tema são raros, apesar da quantidade de dados gerados pela pesquisa estrangeira. Estes dados abrangem informações sobre a correlação entre CS e habilidades ToM e sintomas da esquizofrenia ${ }^{19,20-22}$, bem como sobre a presença de alterações dessas habilidades, tanto em indivíduos suscetíveis mas não diagnosticados como esquizofrênicos ${ }^{24-26}$, como em episódios psicóticos e nos períodos intercríticos do transtorno ${ }^{27,28}$. Outro campo de pesquisa pouco explorado, mas já em início de desenvolvimento, é o dos estudos a respeito do impacto do uso de antipsicóticos sobre a CS e as habilidades ToM.

A compreensão do funcionamento social de esquizofrênicos em um nível cognitivo pode ser útil para o desenvolvimento de técnicas terapêuticas eficazes e de fácil aplicabilidade em ambientes hospitalares e ambulatoriais que possam ser objetivamente medidas.

Frith $^{19}$, em 1992, levantou pela primeira vez a questão da relação entre os sintomas da esquizofrenia e os prejuízos das habilidades ToM. O autor sugeriu que os pacientes com predomínio de sintomas das dimensões negativa e desorganizada teriam comprometimento da capacidade de representar os estados mentais dos outros e de si mesmos, enquanto os pacientes paranoides apresentariam apenas comprometimento da capacidade de representar os estados mentais dos outros. Hardy-Baylé, em $1994^{20}$, argumentou que o prejuízo das habilidades ToM na esquizofrenia apenas refletiria o déficit executivo desses pacientes, de forma que alterações nas habilidades ToM seriam proporcionais à gravidade da síndrome disexecutiva. Como consequência, pacientes paranoides sem sintomas da dimensão desorganizada não teriam déficits de habilidades ToM.

Muitos estudos propuseram-se a correlacionar as alterações das habilidades ToM em esquizofrênicos e os sintomas da esquizofrenia, testando os modelos propostos por Frith e por Hardy-Baylé. Brune $^{29}$, revisando o assunto, concluiu que os dados disponíveis parecem confirmar a hipótese de que os pacientes com predomínio de sintomas negativos e de desorganização têm piores pontuações na testagem de habilidades ToM do que pacientes com predomínio de sintomas positivos. O modelo de Hardy-Baylé parece confirmar-se apenas parcialmente, já que muitos estudos não sustentam sua hipótese de que pacientes paranoides sem extensa sintomatologia desorganizada não apresentariam prejuízos de habilidades ToM.

Em virtude de ainda não estar estabelecido se as habilidades ToM constituem um módulo cognitivo independente no cérebro por funcionarem em profunda consonância com as habilidades cognitivas gerais, o estudo dos prejuízos de habilidades ToM em esquizofrênicos deve controlar as variáveis cognitivas "não ToM" que possam interferir nos resultados. Déficits de atenção e executivos e baixos escores de inteligência objetivamente influenciam a testagem das habilidades ToM, mas a comparação de indivíduos normais com esquizofrênicos no que tange à performance em testes que avaliam tais habilidades continua favorecendo significativamente controles saudáveis quando são controlados os fatores relacionados à cognição ${ }^{29,30}$.

Uma meta-análise realizada recentemente por Sprong et al. ${ }^{30}$ sobre ToM na esquizofrenia avaliou 29 estudos e demonstrou que a performance de pacientes esquizofrênicos nas tarefas empregadas para mensuração de habilidades ToM foi significativamente inferior que a de controles saudáveis. Além disso, variáveis como quociente de inteligência (QI), gênero e idade não afetaram significativamente esse resultado apesar de outras importantes variáveis, como uso de medicação e duração e gravidade da doença, não terem podido ser examinadas por falta de informação em muitos dos estudos incluídos.

Até o momento apenas dois estudos escritos em inglês avaliaram o impacto do uso de antipsicóticos sobre tarefas que avaliem habilidades ToM em pacientes portadores de transtorno do espectro da esquizofrenia (TEE) ${ }^{31,32}$. A Tabela 1 resume as principais características metodológicas de cada um. 
Tabela 1 - Principais caracteristicas dos estudos incluidos

\begin{tabular}{|c|c|c|c|c|c|c|}
\hline Autor & $\begin{array}{l}\text { População } \\
\text { estudada }\end{array}$ & Tipo de estudo & Drogas & Avaliação ToM & $\begin{array}{l}\text { Testagem } \\
\text { cognitiva } \\
\text { não ToM }\end{array}$ & $\begin{array}{c}\text { Outros } \\
\text { instrumentos }\end{array}$ \\
\hline $\begin{array}{l}\text { Savina \& } \\
\text { Beninger }^{32}\end{array}$ & $\begin{array}{l}77 \text { pacientes } \\
\text { esquizofrênicos e } \\
7 \text { portadores de } \\
\text { transtomo } \\
\text { esquizoafetivo, } \\
\text { diagnosticados } \\
\text { pelo DSM-IV; } \\
24 \text { controles } \\
\text { voluntários }\end{array}$ & $\begin{array}{l}\text { Estudo } \\
\text { comparativo } \\
\text { aberto }\end{array}$ & $\begin{array}{l}\text { Antipsicóticos } \\
\text { tipicos, } \\
\text { clozapina, } \\
\text { olanzapina, } \\
\text { risperidona. } \\
\text { Pacientes } \\
\text { podiam estar } \\
\text { recebendo } \\
\text { outras drogas } \\
\text { psicotrópicas, } \\
\text { exceto } \\
\text { anticolinérgicos. }\end{array}$ & $\begin{array}{l}\text { Tarefas de crença } \\
\text { de } 1^{2} \text { ordem: } \\
\text { sequência de } \\
\text { figuras } \\
\text { descrevendo } \\
\text { relações } \\
\text { mecânicas, } \\
\text { comportamentais } \\
\text { e intencionais } \\
\text { (requerendo } \\
\text { atribuição de } \\
\text { estados mentais); } \\
\text { Tarefas de falsa } \\
\text { crença de } 2^{2} \\
\text { ordem: histórias } \\
\text { que medem a } \\
\text { capacidade de } \\
\text { compreensão de } \\
\text { que um } \\
\text { protagonista } \\
\text { sustenta uma } \\
\text { falsa crença } \\
\text { envolvendo outro } \\
\text { personagem (Ice- } \\
\text { cream Van e } \\
\text { Family) } \\
\text { Teste Faux Pas }\end{array}$ & MMSE & BPRS \\
\hline $\begin{array}{l}\text { Mizrahi et } \\
\text { al. }^{31}\end{array}$ & $\begin{array}{l}71 \text { pacientes } \\
\text { portadores de } \\
\text { esquizofrenia, } \\
\text { transtomo } \\
\text { esquizofreniforme } \\
\text { e transtomo } \\
\text { esquizoafetivo na } \\
\text { fase transversal; } \\
17 \text { pacientes } \\
\text { portadores de } \\
\text { esquizofrenia, } \\
\text { transtomo } \\
\text { esquizoafetivo e } \\
\text { transtomo } \\
\text { esquizofreniforme } \\
\text { na fase } \\
\text { longitudinal. }\end{array}$ & $\begin{array}{l}\text { Estudo } \\
\text { transversal em } \\
\text { uma } 1^{2} \text { fase e } \\
\text { longitudinal } \\
\text { posterionmente. }\end{array}$ & $\begin{array}{l}\text { Fase transversal: } \\
\text { antipsicóticos } \\
\text { atipicos e típicos } \\
\text { Fase } \\
\text { longitudinal: } \\
\text { risperidona, } \\
\text { olanzapina, } \\
\text { loxapina e } \\
\text { clozapina. }\end{array}$ & Hinting Task & WAIS & $\begin{array}{l}\text { MINI, } \\
\text { PANSS }\end{array}$ \\
\hline
\end{tabular}

O estudo de Mizrahi et al. ${ }^{31}$ avaliou o efeito do tratamento antipsicótico sobre a ToM nos pacientes com TEE tanto transversal quanto longitudinalmente. Na fase transversal do estudo, foi avaliada a performance em uma tarefa envolvendo habilidades ToM de pacientes portadores de TEE que estavam em uso de antipsicóticos. Na fase longitudinal, pacientes com TEE, a maioria deles em primeiro surto, tiveram sua performance na mesma tarefa avaliada ao longo de 6 semanas a partir da introdução de antipsicóticos. Na fase transversal, os autores encontraram escores da tarefa ToM semelhantes aos encontrados em uma população avaliada anteriormente por Corcoran et al. ${ }^{15}$. Além disso, esses escores correlacionaram-se significativamente com os sintomas negativos e gerais avaliados pela Positive and Negative Syndrome Scale (PANSS), mas não com os sintomas positivos, o que está de acordo com a proposta inicial de Frith $^{19}$. Longitudinalmente houve melhora das pontuações da tarefa ToM ao longo das 6 semanas de tratamento. Além disso, os escores dos pacientes na baseline não estavam associados significativamente com os sintomas positivos, mas sim com os sintomas negativos e gerais. Embora Mizrahi et al. ${ }^{31}$ tenham sido os pioneiros a examinar Iongitudinalmente os efeitos do tratamento antipsicótico nas habilidades ToM nos TEE, seu estudo 
apresenta uma série de limitações metodológicas: ausência de grupos-controle, utilização de apenas uma tarefa ToM e de apenas uma testagem cognitiva não ToM, amostra muito pequena na fase longitudinal e predomínio de pacientes do sexo masculino nas duas fases ( $85 \%$ na fase transversal e $76 \%$ na fase longitudinal).

Savina \& Beninger ${ }^{32}$ compararam o impacto do uso de antipsicóticos típicos e atípicos em pacientes portadores de TEE sobre a performance em três tipos de tarefas ToM de complexidade variável com o desempenho de um grupo de voluntários saudáveis nas mesmas tarefas. Os resultados mostraram melhores pontuações em tarefas ToM por indivíduos em uso de olanzapina e clozapina e piores pontuações nas mesmas tarefas por indivíduos usando antipsicóticos típicos e risperidona. Os autores propõem que tais resultados possam correlacionar-se à ativação de c-fos e consequente liberação de dopamina (DA) pela olanzapina e clozapina (mas não pela risperidona e típicos) no córtex pré-frontal, principal sítio anatômico das habilidades ToM. Todavia, outras funções cognitivas são melhoradas pelo aumento da DA frontocortical e não apenas aquelas relacionadas à cognição social, como foi demonstrado anteriormente ${ }^{33-35}$. Além disso, o estudo de Savina $\&$ Beninger $^{32}$ utilizou apenas o Mini Exame do Estado Mental para o controle cognitivo não ToM, sendo necessárias medidas mais precisas e específicas da atividade cognitiva geral para que tais conclusões possam ser consideradas com maior segurança. Outras limitações do seu estudo incluem a possibilidade de os pacientes estarem usando outras drogas além dos antipsicóticos, desenho aberto e ausência de grupo placebo.

\section{CONCLUSÕES}

A esquizofrenia é um transtorno mental que cursa com severo comprometimento da capacidade de interagir socialmente de forma adequada e funcional. Tal incapacidade pode estar relacionada a alterações de circuitos cerebrais especificamente relacionados com a CS, circuitos esses que têm sido extensivamente estudados, principalmente nos transtornos do espectro do autismo e na esquizofrenia. Uma das linhas de pesquisa da CS é a que enfoca a capacidade de indivíduos saudáveis de inferirem estados mentais dos outros, também chamada de teoria da mente. Apesar da existência de várias tarefas verbais e não verbais disponíveis para a medida das habilidades ToM, elas não foram especificamente desenhadas para a aplicação em pacientes esquizofrênicos e não foram validadas ainda. Para serem realizadas, essas tarefas recrutam circuitos relacionados à cognição geral, em maior ou menor intensidade, tornando difícil a interpretação de muitos dos resultados obtidos até o momento com a pesquisa das habilidades ToM em esquizofrênicos. Recentemente, uma tentativa de identificar fatores cognitivos separáveis no estudo da esquizofrenia foi realizada em uma iniciativa do National Institute of Mental Health dos Estados Unidos denominada Measurement and Treatment Research to Improve Cognition in Schizophrenia ${ }^{18}$. Este estudo conclui que atualmente ainda não é possível determinar a extensão com que a CS pode ser considerada um domínio cognitivo separado. Para esclarecer essa questão, os autores recomendam, além da elaboração de instrumentos padronizados para a mensuração da CS, a verificação da possibilidade de a CS ser um domínio cognitivo específico mediante o emprego da análise fatorial.

Os estudos relacionando habilidades ToM e antipsicóticos são poucos e suscetíveis a várias críticas em relação a sua metodologia, razão pela qual devem ser interpretados com cautela.

O crescente interesse pelo estudo da CS e das habilidades ToM poderá disponibilizar dados a serem utilizados no desenvolvimento de programas adjuvantes à farmacoterapia e que se relacionem não só ao manejo dos sintomas psicóticos como, quem sabe, à prevenção do desenvolvimento do transtorno em indivíduos suscetíveis.

\section{REFERÊNCIAS}

1. Pinkham AE, Penn DL, Perkins DO, Lieberman J. Implications for the neural basis of social cognition for the study of schizophrenia. Am J Psychiatry. 2003; 160:815-24.

2. Barret L, Henzi P. The social nature of primate cognition. Proc R Soc B. 2005; 272:1865-75. 
3. Wang AT, Lee S, Sigman M, Dapretto M. Reading affect in the face and voice: neural correlates of interpreting communicative intent in children and adolescents with autism spectrum disorders. Arch Gen Psychiatry. 2007;64(6):698-708.

4. Oberman LM, Ramachandran VS. The simulating social mind: the role of the mirror neuron system and simulation in the social and communicative deficits of autism spectrum disorders. Psychol Bull. 2007;133(2):310-27.

5. Burns J. The social brain hypothesis of schizophrenia. World Psychiatry. 2006;5(2):77-81.

6. Premack D, Woodruff G. Does the chimpanzee have a theory of mind? Behav Brain Sci. 1978; 1:515-26.

7. De Waal FBM. Peace making among primates. Cambridge: Harvard University Press; 1989.

8. Brunet-Gouet E, Decety J. Social brain dysfunctions in schizophrenia: a review of neuroimaging studies. Psychiatry Research: Neuroimaging. 2006; 148: 75-92.

9. Saper CB, Iversen S, Frackowiack R. Integration of sensory and motor function: the association areas of the cerebral cortex and the cognitive capabilities of the brain. 4th ed. In: Kandel ER, Schwartz JH, Jessel TM, eds. Principles of neural Science. New York: McGraw-Hill; 2000. p. 349-80.

10. Wimmer $\mathrm{H}$, Perner J. Beliefs about beliefs: representation and constraining function of wrong beliefs in young childrens understanding of deception. Cognition. 1983;13(1):103-28.

11. Winner E, Brownell H, Happe F, Blum A, Pincus D. Distinguishing lies from jokes: theory of mind deficits and discourse interpretation in right hemisphere brain-damaged patients. Brain Lang. 1998;62(1):89-106.

12. Muris $P$, Steerneman $P$, Meesters $C$, et al. The TOM test: a new instrument for assessing theory of mind in normal children and children with pervasive developmental disorders. J Autism Dev Disord. 1999;29(1):67-80.

13. Baron-Cohen S, O'Riordan M, Stone V, Jones R, Plaisted K. Recognition of faux pas by normally developing children and children with Asperger syndrome or high-functioning autism. J Autism Dev Disord. 1999;29(5):407-18.

14. Stone V, Baron-Cohen S, Knight R. Frontal lobe contributions to theory of mind. Journal of Cognitive Neuroscience. 1998; 10:640-56.

15. Corcoran R, Mercer G, Frith CD. Schizophrenia, symptomatology and social inference: investigating "Theory of Mind" in people with schizophrenia. Schizophr Res. 1995; 17:05-13.

16. Baron-Cohen S, Jollife T, Mortimore C, Robertson M. Another advanced test of theory of mind: Evidence from very high-functioning adults with autism or Asperger Syndrome. J Child Psychol Psychiat. 1997;38:813-22.

17. Baron-Cohen S, Wheelwright S, Hill J, Raste Y, Plumb I. The "Reading the mind in the eyes" test revised version: a study with normal adults, and adults with Asperger syndrome or high-functioning autism. J Child Psychol Psychiat. 2001;42(2):241-51.

18. Nuechterlein KH, Barch DM, Gold J M, Goldberg TE, Green MF, Heaton RK. Identification of separable cognitive factors in schizophrenia. Schizophr Res. 2004; 72:29-39.

19. Frith CD. The Cognitive neuropsychiatry of schizophrenia. Hove: Lawrence Erlbaum Associates; 1992.

20. Hardy-Baylé MC, Sarfati Y, Passerieux C. The cognitive basis of disorganization symptomatology in schizophrenia and its clinical correlates: toward a pathogenetic approach to disorganization.

Schizophr Bull. 2003;29(3):459-71.

21. Sarfati Y, Hardy-Baylé MC. How do people with schizophrenia explain the behaviour of others? A 
study of theory of mind and its relationship to thought and speech disorganization in schizophrenia. Psychol Med. 1999;29(3):613-20.

22. Sarfati $Y$, Hardy-Baylé MC, Brunet E, Widlocher D. Investigating theory of mind in schizophrenia: influence of verbalization in disorganized and non-disorganized patients. Schizophr Res. 1999;37(2): 183-90.

23. Frith CD, Blakemore S, Wolpert DM. Explaining the symptoms of schizophrenia: abnormalities in the awareness of action. Brain Research. Brain Research Reviews. 2000;31:357-63.

24. Pickup GJ. Theory of mind and its relation to schizotypy. Cognit Neuropsychiatry. 2006; 11(2): 177-92.

25. Jahshan CS, Sergi MJ. Theory of mind, neurocognition, and functional status in schizotypy. Schizophr Res. 2006; 89(1-3):278-86.

26. Langdon R, Coltheart M. Mentalizing, schizotypy and schizophrenia. Cognition. 1999;71:43-71.

27. Drury VM, Robinson EJ, Birchwood M. Theory of mind skills during an acute episode of psychosis and following recovery. Psychological Medicine. 1998;28:1101-12.

28. Herold R, Tényi T, Lenard K, Trixler M. Theory of mind deficit in people with schizophrenia during remission. Psychological Medicine. 2002;32:1125-9.

29. Brune M. "Theory of Mind" in schizophrenia: a review of the literature. Schizophr Bull. 2005;31(1):21-42.

30. Sprong M, Schothorst P, Vos E, Hox J, Van Engeland H. Theory of mind in schizophrenia. Meta-analysis. Br Jour Psych. 2007;191:5-13.

31. Mizrahi R, Korostil M, Starkstein SE, Zipursky RB, Kapur S. The effect of antipsychotic treatment on theory of mind. Psychol Med. 2007;37:595-601.

32. Savina I, Beninger RJ . Schizophrenic patients treated with clozapine or olanzapina perform better on theory on mind tasks than those treated with risperidone or typical antipsychotic medications. Schizophr Res. 2007;94(1-3):128-38.

33. Meltzer HY, Mcgurk SR. The effects of clozapine, risperidone and olanzapina on cognitive function in schizophrenia. Schizophr Bull. 1999;25(2):233-55.

34. Deutch AY, Duman RS. The effects of antipsychotic drugs on Fos protein expression in the prefrontal cortex: cellular localization and pharmacological chacterization. Neuroscience. 1996; 70(2):377-89.

35. Friedman JI, Temporini H, Davis KL. Pharmacologic strategies for augmenting cognitive performance in schizophrenia. Biol Psychiatry. 1999;45(1):01-16.

\section{团 Correspondência}

Helio Tonelli

Av. Cândido de Abreu, 526/201

CEP 80.240-140, Bairro Água Verde, Curitiba, PR

Tel.: (41) 3252.6988, (41) 3243.6339, (41) 9106.0693

E-mail: hatonelli@terra.com.br

Recebido em 28/08/2007.

Aceito em 15/10/2007.

Não foram declarados conflitos de interesse associados à publicação deste artigo. 